\title{
Results of L3 BGO Calorimeter Calibration using an RFQ accelerator ${ }^{1}$
}

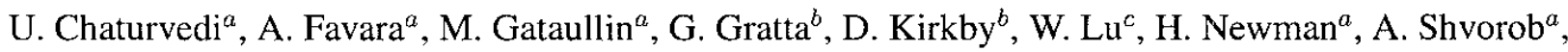 \\ C. Tully ${ }^{d}$, R. Zhu ${ }^{a}$ \\ ${ }^{a}$ Physics Department, California Institute of Technology, Pasadena, CA 91125 \\ ${ }^{b}$ Now at Stanford University, Stanford, CA 94305 \\ Now at Jet Propulsion Laboratory, Pasadena, CA 91109 \\ ${ }^{d}$ Physics Department, Princeton University, Princeton, NJ 08544
}

\begin{abstract}
A novel calibration system based on a radio-frequencyquadrupole (RFQ) accelerator has been installed in the L3 experiment. Radiative capture of $1.85 \mathrm{MeV}$ protons from the RFQ accelerator in a lithium target produces a high-intensity flux of $17.6 \mathrm{MeV}$ photons which are used to calibrate 11,000 crystals of the L3 BGO calorimeter. In this paper, we present results of the first fully successful RFQ run, taken in November 1997. We achieved a calibration precision of $0.6 \%$ in the barrel and $0.7 \%$ in the endcaps of the $\mathrm{L} 3 \mathrm{BGO}$ calorimeter. The RFQ-97 calibration proved to be the best one of all calibration techniques used in the L3 experiment.
\end{abstract}

\section{INTRODUCTION}

L3 is one of the four experiments operating at the LEP $e^{+} e^{-}$collider at CERN. The L 3 electromagnetic calorimeter, composed of bismuth germanate (BGO) crystals, is one of the key parts of the detector. The crystals are arranged in two endcaps (each of 1527 crystals) and two half-barrels (7680 crystals combined) [1]. In order to maximize the discovery potential of the detector one of the technical performance goals of L3 is to measure electron and photon energies with $1 \%$ resolution over the energy range from a few $\mathrm{GeV}$ up to $100 \mathrm{GeV}$. This requires a precision calibration of the electromagnetic calorimeter, consisting of the determination of calibration constants for each crystal so that the raw ADC channel values may be converted to the energy deposited in the crystal.

Since the first BGO calibrations performed at test beams prior to the startup of LEP in 1987\%8, where resolutions of $\simeq 1 \%$ were seen, many calibration techniques have been applied in order to obtain the best results. Among these, the Radiofrequency Quadrupole (RFQ) calibration system [2], shown together with the L3 detector in Figure 1, developed by Caltech in collaboration with the World Laboratory, has proven to be the most precise.

We present here results of the November 1997 RFQ run, which represented a major step forward: a calibration precision of $0.6 \%$ and $0.7 \%$ was achieved for crystals in the barrel and in the endcaps, respectively, thus, recovering an intrinsic resolution comparable to that achieved in test beam 10 years ago.

\footnotetext{
${ }^{1}$ This work was supported in part by the U.S. Department of Energy Grant No. DE-FG03-92-ER40701.
}

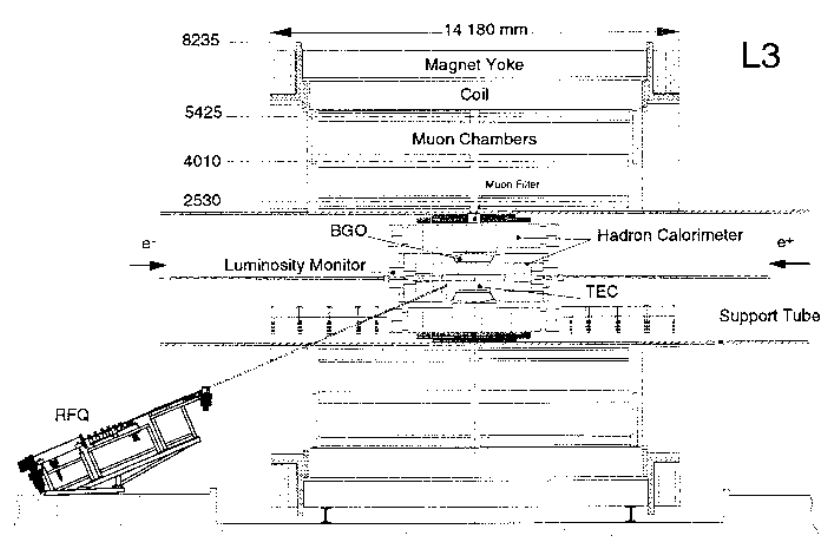

Figure 1: A side view of the RFQ system installed in the $\mathrm{L} 3$ detector.

\section{L3 RFQ CALIBRATION SYSTEM}

The L3 RFQ calibration system (Figure 2) consists of the following components:

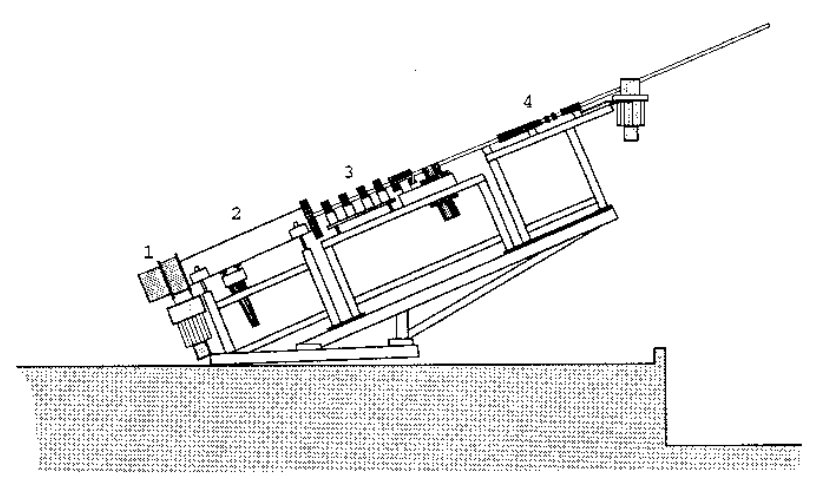

Figure 2: The RFQ system: 1) ion source; 2) RFQ ion accelerator; 3) steering and focusing magnets; 4 ) beam neutralizer.

- A $30 \mathrm{keV}$ RF-driven ( $2 \mathrm{MHz}$ ) volume $H^{-}$ion source and a low-energy beam transport;

- A $1.85 \mathrm{MeV}$ RFQ (425 MHz) accelerator, which can provide an $H^{-}$current of $7.5 \mathrm{~mA}$;

- A high-energy beam transport, consisting of quads and an $x y$-steering magnet; 
- A beam neutralizer $\left(H^{-} \rightarrow H^{o}+e^{-}\right)$, consisting of a $1 \mathrm{~m}$ long $N_{2}$ gas cell, at a typical pressure of $5 \cdot 10^{-4}$ Torr corresponding to a neutralization efficiency of $55 \%$;

- A $10 \mathrm{~m}$ long beam pipe, equipped with a star-cell ion pump (20 LPS) and a non-evaporable getter ribbon pump (3 LPS);

- A water-cooled $\mathrm{LiH}$ target, mechanically sealed with a thin Ta foil, mounted on the end of a $10 \mathrm{~m}$ long beam pipe;

- Calibration data acquisition and readout systems.

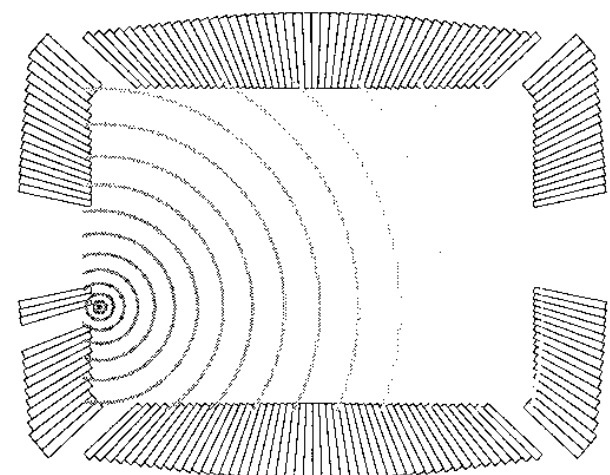

Figure 3: A side view of the BGO calorimeter with concentric circles representing the photon flux originating from the RFQ target.

The RFQ system is shielded from the residual fringe magnetic field outside the L3 magnetic door. The RFQ calibration technique uses a pulsed $\mathrm{H}^{-}$beam from the RFQ accelerator to bombard a lithium target installed inside the BGO calorimeter. After focusing and steering, the beam is neutralized to allow it to pass undisturbed through the magnetic field of L3. Radiative capture of the protons

$$
p+{ }_{3}^{7} \mathrm{~L} i \rightarrow{ }_{4}^{8} \mathrm{~B} e+\gamma
$$

produces 17.6 MeV photons that are used to calibrate the calorimeter. Figure 3 shows the target location with respect to the BGO crystals and the propagation of the calibration photon flux. The RFQ accelerator is synchronized to the BGO readout system, so that the calibration signal from the photon flux receives the same integration gate as the data coming from the LEP $e^{+} e^{-}$collider.

The photon energy spectrum of each crystal is histogramed in a readout memory. A veto on the energy deposition in the eight adjacent crystals is implemented to reject photons with energy shared between two crystals. The components of the RFQ system are described in more detail in [3].

\section{CAlibration in situ}

\section{A. "RFQ Only" Calibration}

The RFQ-97 run was taken from November 11 to November 15, 1997. With an average DAQ rate of $70 \mathrm{~Hz}$, we recorded about 9 million triggers. The photon rate is characterized by the photon occupancy, defined as the fraction of triggers with energy deposition in one crystal larger than $14 \mathrm{MeV}$. The occupancy differs from crystal to crystal due to the varying location of the crystals relative to the Lithium target, and the material in the L3 tracker (TEC) end-flanges. The typical occupancy in the barrel region was $0.08 \%$ for the half-barrel nearest to the target (Figure 4), and $0.03 \%$ for the half-barrel on the far side. A typical photon energy

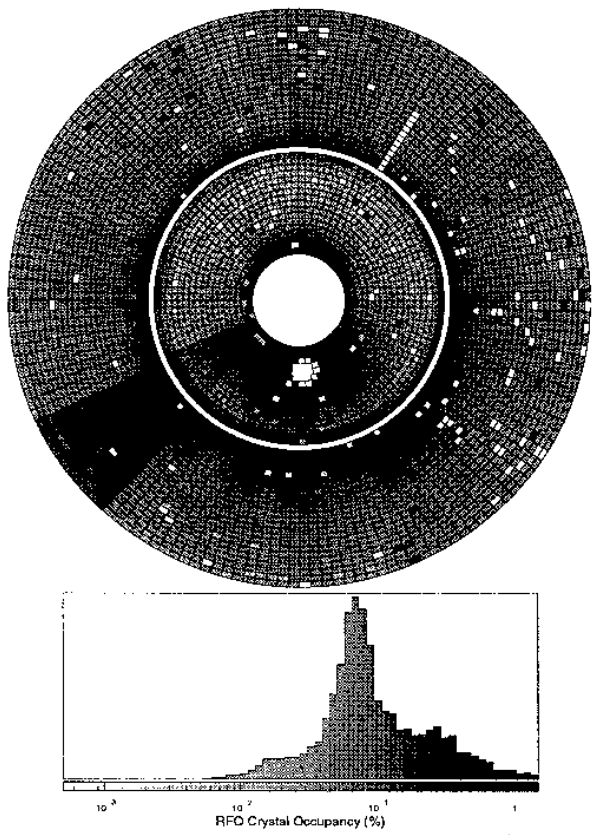

Figure 4: The photon occupancy for the near half-barrel (outer rings) and endcap (inner rings). The central hole in the endcap is for the passage of the LEP beam pipe. The smaller hole located just below is for the RFQ beam pipe and target insertion.

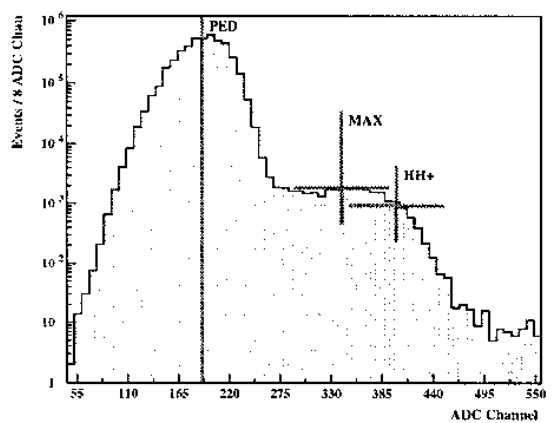

Figure 5: A typical photon energy spectrum shown with the definition of the $\mathrm{HH}^{+}$calibration point.

spectrum deposited in a BGO crystal is shown in Figure 5. For each crystal the "RFQ Only" calibration constant, C.C. (kev/ADC Channel), is then obtained by

$$
\text { C.C. }=\frac{E_{H H^{+}}}{H H^{+}-\text {Pedestal }}, E_{H H^{+}} \approx 17.6 \mathrm{MeV}
$$

where the " $H H^{+}$" edge is the specific feature used for calibration and is defined as the point half-way below and to the right of the calibration signal peak. The sharpness of this 


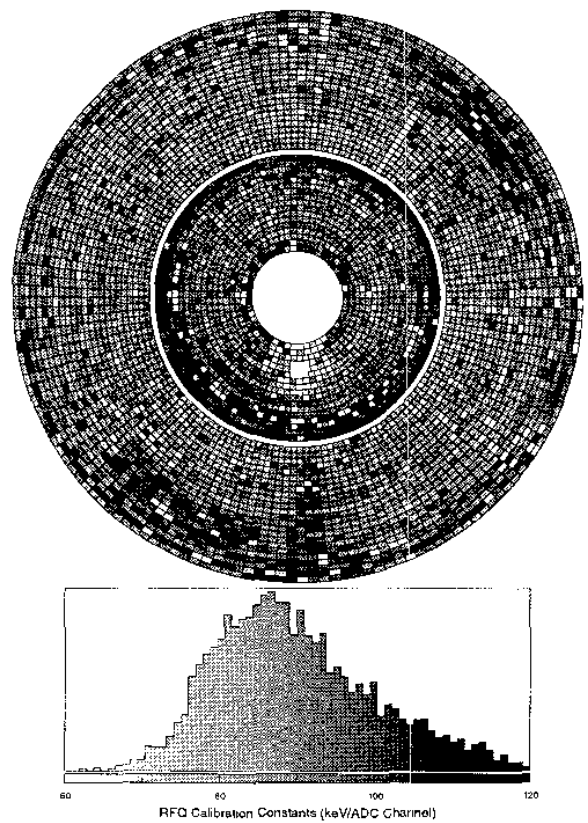

Figure 6: The RFQ calibration constants for the near half-barrel (outer rings) and endcap (inner rings). The distribution of the calibration constants is shown in the lower histogram.

falling edge ensures that the $\mathrm{HH}^{+}$point is the least sensitive to a variety of possible systematic effects such as the noise-level, the incident photon angle and the amount of material in front of the crystals [3]. The values of the calibration constants determined for the BGO crystals are shown in Figure 6. A typical calibration constant value is $90 \mathrm{keV} / \mathrm{ADC}$ channel.

The "RFQ Only" calibration gives a Bhabha peak resolution of $2.5 \%$, largely due to the extrapolation from the energy scale of the calibration $(17 \mathrm{MeV})$ up to the $90 \mathrm{GeV}$ beam energy range. The slight loss of resolution over this large range is a result of the BGO non-linearity due to electromagnetic shower leakage, light collection non-uniformity and energy loss in the material between the BGO crystals.

\section{B. "RFQ+Bhabha" Method}

At $e^{+} e^{-}$colliders, such as LEP, Bhabha scattering $\left(e^{+} e^{-} \rightarrow e^{+} e^{-}(\gamma)\right)$ produces electrons of energy close to that of the beam. The beam energy known with a very good precision is widely used as a high-energy calibration point.

To improve the "RFQ Only" calibration, the "RFQ+Bhabha" method was developed. The "RFQ+Bhabha" method uses the "RFQ Only" calibration as an inter-calibration. The energy spectrum from high-energy electrons from the Bhabha scattering process is then systematically applied to correct for geometrical effects and the non-linearity of the calorimeter energy response. The correction factor for a given crystal is obtained through:

$$
C . F .=\frac{1}{\sum_{i=1}^{N_{\text {e. }}} w_{i}} \sum_{i=1}^{N_{\text {e.t }}} \frac{E_{\text {beam }}}{E_{i}} w_{i}
$$

where:
1. $N_{e e}$ is the number of selected BGO "bumps" containing this crystal in the $3 \times 3$ crystal matrix centered on the crystal with the maximum energy deposition ;

2. $E_{\text {beam }}$ is the beam energy;

3. $E_{i}$ is the energy of the $i$ th bump computed using ADC values and the calibration constants from the "RFQ Only" method;

4. $w_{i}$ is the weight assigned to the $i$ th event for the crystal, which we put to be equal to the ratio of the energy deposited in the crystal to the total energy deposited in the $3 \times 3$ crystal matrix. (Both energies were computed using the calibration constants from the "RFQ Only" method and ADC values.)

Special care was taken when a bump contained a dead crystals in its $3 \times 3$ matrix or when it was on the edge of the BGO detector. To evaluate $E_{i}$ and $w_{i}$ for such bumps we used a shower-fitting algorithm, which corrects for bump energy loss due to dead or missing crystals in the $3 \times 3$ matrix.

For each crystal the calibration constant is multiplied by the corresponding correction factor. Then we again computed correction factors for the new calibration constants. We needed to repeat this procedure a few times before the calibration constants converged to stable values. Doing such iterative computation significantly improves calibration precision.

The BGO resolution obtained using the "RFQ+Bhabha" method is significantly better than the resolution obtained with the "RFQ Only" calibration constants. However, we must underline that the precise $\mathrm{BGO}$ inter-calibration done with $\mathrm{RFQ}$ data is the cornerstone of the "RFQ+Bhabha" method. Special studies have shown that the best calibration accuracy obtained using only Bhabha events is more than two times worse than the accuracy achieved using the RFQ inter-calibration.

To increase the Bhabha statistics it was necessary to use the data collected at all LEP beam energies from 1995 to 1997 . To correct for BGO response evolution both in time and in energy we performed careful studies of the calorimeter non-linearity and aging, described in the following section.

\section{Other BGO Calorimeter Studies}

Since the beginning of LEP operation in 1989 it was noticed that the response of the BGO calorimeter drifts with time towards lower values. The decay trend is described quite well with a function

$$
F(t)=\frac{a}{t-t_{0}}+C
$$

with $a, t_{0}$, and $C$ as free parameters. These parameters are different for all four BGO subdetectors ${ }^{3}$, as they were

\footnotetext{
${ }^{2} \mathrm{~A}$ BGO bump refers to a local maximum of energy deposition in the calorimeter and the surrounding region of crystals containing energy.

${ }^{3}$ The BGO detector consists of two half-barrels and two endcaps.
} 
manufactured and installed separately. The evolution plots and fitted functions are shown in Figure 7. As one can see, the decay trend is more pronounced in the endcaps than in the barrel, which is explained by the fact that the endcaps were installed two years later.

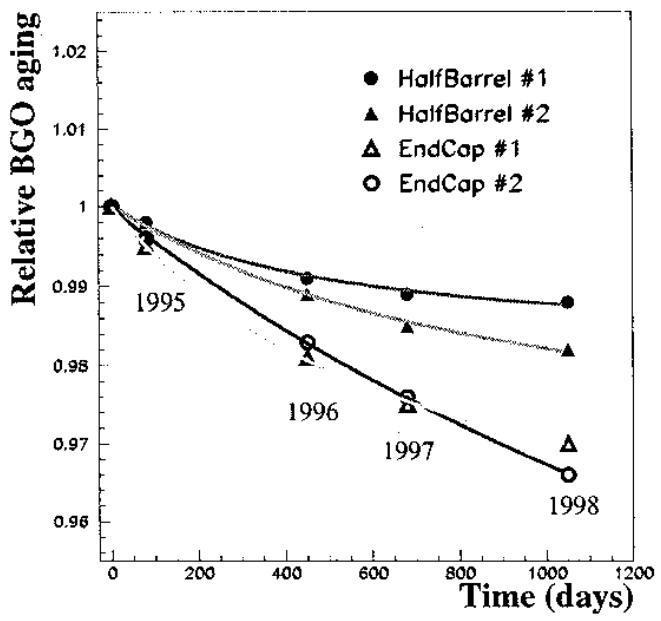

Figure 7: The BGO aging curves obtained with several LEP runs at a fixed beam energy of $45.6 \mathrm{GeV}$.

Another effect which had to be taken into account was BGO non-linearity. During the years 1995-1997, LEP machine gradually increased center-of-mass energy, going from $91 \mathrm{GeV}$ up to $183 \mathrm{GeV}$. We discovered that the BGO response is non-linear with energy. Every year LEP performs at least one calibration run with a $91 \mathrm{GeV}$ center-of-mass energy (" $Z$ peak" runs). To determine the non-linearity function we measured the difference between the Bhabha peak position at an energy higher than $91 \mathrm{GeV}$ and the position of the Bhabha peak obtained with a $91 \mathrm{GeV}$ run performed in the same year. Thus, we were able to separate aging and non-linearity effects. As can be seen in Figure 8, the BGO response shift between the beam energy of $91.36 \mathrm{GeV}$ and that of $45.6 \mathrm{GeV}$ is about $0.6 \%$, i.e. almost the same as the calibration accuracy.

\section{CAlibRation ACCURACY MEASUREMENT}

The BGO calorimeter overall resolution was determined using a large sample of Bhabha events collected in 1998. This sample was not used by the "RFQ+Bhabha" method, and, therefore, the measured overall $\mathrm{BGO}$ energy resolution from this data sample is unbiased. To fit the Bhabha energy spectrum we used the lineshape function obtained by the Crystal Ball experiment given by the following formula [4]

$$
\begin{aligned}
\Psi\left(E_{m} \mid E_{t}, \sigma, A, a, n\right)= & A \exp \left(\frac{-\left(E_{t}-E_{m}\right)^{2}}{2 \sigma^{2}}\right) \\
& \text { if } E_{m}>E_{t}-a \sigma \\
\Psi\left(E_{m} \mid E_{t}, \sigma, A, a, n\right)= & A\left(\frac{n}{a}\right)^{n} \frac{\exp \left(\frac{-a^{2}}{2}\right)}{\left(\frac{E_{t}-E_{m}}{\sigma}+\frac{n}{a}-a\right)^{n}} \\
& \text { if } E_{m} \leq E_{t}-a \sigma
\end{aligned}
$$

where $n$ and $a$ are empirical parameters, $E_{m}$ is a measured energy, $E_{t}$ is the Bhabha peak position and $\sigma$ is the energy

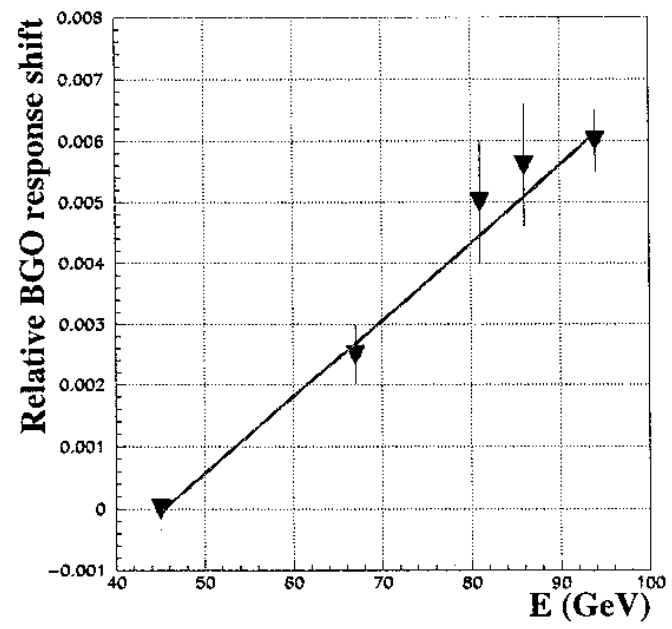

Figure 8: The BGO non-linearity function obtained with LEP runs at different center-of-mass energies.

resolution.

The measured BGO overall resolution is $1.1 \%$ in the barrel and $1.2 \%$ in the endcaps, as shown in Figure 9.

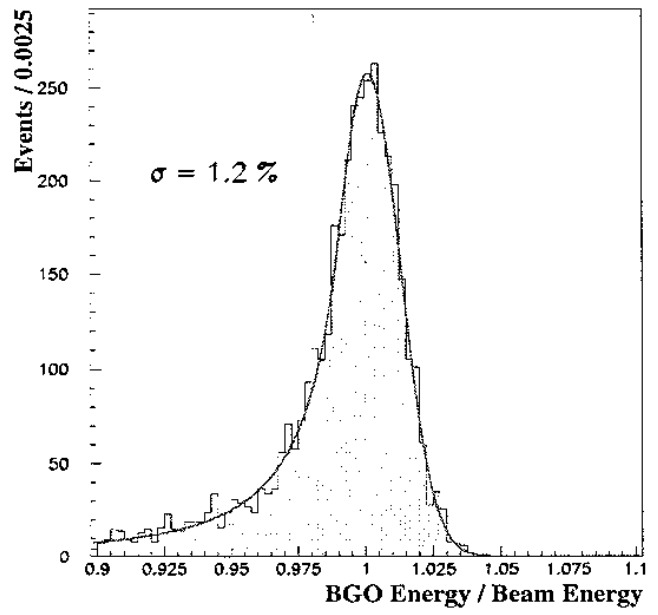

Figure 9: The 1998 endcap Bhabha energy spectrum fitted with the Crystal Ball lineshape function.

Several other factors together with the RFQ calibration accuracy contribute to this resolution. Most notable are the smearing of the Bhabha peak by the radiative events, "intrinsic" detector resolution effects, such as electromagnetic shower fluctuation and shower leakage, and the accuracy of the temperature determination.

The light response of a $\mathrm{BGO}$ crystal is strongly correlated to the crystal temperature with a coefficient of $-1.55 \% /{ }^{\circ} \mathrm{C}$. The temperature at the front and the back side of the $\mathrm{BGO}$ calorimeter is constantly monitored by 1280 sensors. On the basis of this data, a two dimensional fit is performed yielding the temperature of each crystal. A temperature correction is then applied further to the measured BGO energy. The estimated errors on the temperature measurement contribute $0.6 \%$ to the overall BGO resolution in the barrel and $0.8 \%$ in the endcaps. 
The error on the measured temperature is smaller in the barrel due to its more stable cooling system and better performance of the temperature sensors.

The contribution of the Bhabha radiative smearing and the intrinsic detector resolution effects to the overall resolution was estimated using a large sample of Monte Carlo Bhabha events, which were processed through a detailed simulation of the ideal L3 detector. "Ideal" in this case means that the calibration constants and the temperature measurements of all BGO crystals are assumed to be perfectly known in the simulation. However, other detector imperfections and electromagnetic shower fluctuations are accurately simulated. The Bhabha radiative smearing and the intrinsic $\mathrm{BGO}$ resolution were estimated to contribute in total $0.8 \%$ in the barrel and $0.6 \%$ in the endcaps.

Subtracting in quadrature the "radiative", intrinsic and temperature resolutions from the overall $\mathrm{BGO}$ resolution, we obtained the calibration accuracy of $0.6 \%$ for the barrel calorimeter and $0.7 \%$ for the endcaps. Factors contributing to the overall BGO resolution are summarized in Table 1.

Table 1

The BGO resolution achieved with the RFQ Calibration.

\begin{tabular}{|c|c|c|}
\hline & Barrel & Endcaps \\
\hline $\begin{array}{c}\text { "Radiative" error } \\
\text { + Intrinsic }\end{array}$ & $0.8 \%$ & $0.6 \%$ \\
\hline Temperature error & $0.6 \%$ & $0.8 \%$ \\
\hline Calibration error & $0.6 \%$ & $0.7 \%$ \\
\hline \hline Overall & $1.1 \%$ & $1.2 \%$ \\
\hline
\end{tabular}

\section{CONCLUSION}

By analyzing data from the RFQ-97 calibration run, we have obtained a significantly more precise BGO calorimeter calibration than was previously available. This calibration is now in use for the L.3 data reconstruction and physics analyzes, both for a wide range of new particle searches and for the study of electroweak and radiative QED processes at LEP2.

We achieved a calibration precision of $0.6 \%$ in the barrel and $0.7 \%$ in the endcaps of the L3 BGO calorimeter. The RFQ-97 calibration, including high statistics and new analysis, has been shown to provide the highest resolution since the BGO calorimeter was installed in LEP. The RFQ calibration, and the new calibration analysis, will be used throughout the remainder of the LEP2 physics program, up to center-of-mass energies of approximately $200 \mathrm{GeV}$ by the year 2000 .

\section{REFERENCES}

[1] L3 Collab., B. Adeva et al., Nucl. Instr. and Meth. A289 (1990) pp. 35-102.

[2] R. Y. Zhu et. al., Nuclear Physics B (Proc. Suppl.) 44 (1995) pp. 109-115.

[3] H. Ma et. al., Nucl. Instr. and Meth. A274 (1989) pp. 113-128.

[4] I. C. Brock "Mn_Fit, A Fitting and Plotting package Using MINUIT”, L3 Note 918. 